\title{
金绿宝石 $\mathrm{Al}_{2} \mathrm{BeO}_{4}$ 的 Raman 散射谱
}

\author{
贾惟义 郑庆荣 商玉生 王彦云 姚振益 何寿安 周赫田 刘莉玲 \\ (中国科学院物理研究所, 北京) \\ (北京大学物理系)
}

金绿宝石 $\mathrm{Al}_{2} \mathrm{BeO}_{4}$ 具有橄榄石 $(\mathrm{MgFe})_{2} \mathrm{SiO}_{4}$ 结构, 属正交晶系, 空间群为 $P_{n m a}-D_{2 h}^{16[1]}$. 由于地质学和矿物学上的兴趣, 对橄榄石和 $\mathrm{Mg}_{2} \mathrm{SiO}_{4}$ 的晶格振动性质曾进行过相当多的研 究 ${ }^{[2-4]}$. 对于金绿宝石 $\mathrm{Al}_{2} \mathrm{BeO}_{4}$ 晶格振动性质却研究得较少. 近年来发现, $\mathrm{Al}_{2} \mathrm{BeO}_{4}$ 是一种 很好的激光工作基质材料. 用掺 $\mathrm{Cr}^{3+}$ 的 $\mathrm{Al}_{2} \mathrm{BeO}_{4}$ 晶体制成了可调谐固体声子态激光器 ${ }^{[5]}$. 这 种激光器的工作能级是 $\mathrm{Cr}^{3+}$ 离子的电声子边带. 而这种电声子边带的频谱相应着 $\mathrm{Al}_{2} \mathrm{BeO}_{4}$ 的 一级或多级声子谱. 因此研究 $\mathrm{Al}_{2} \mathrm{BeO}_{4}$ 的晶格振动谱就有特别的意义.

文献中对橄榄石的振动模的群论分析结果有一些细节上的不一致 ${ }^{[2-4]}$. 这里我们根据金 绿宝石的结构资料 ${ }^{[1]}$,对其振动模做了分析. 采用了两种方法：（1）商群分析法. 利用空间群 $D_{2 h}^{16}$ 各种对称操作下位置保持不变的原子数目, 对 $K=0$ 点, 即布里洲区中心, 分析振动模的对 称性质 ${ }^{[6]}$; (2) 位置群分析法 ${ }^{[7]}$. 在晶体中选取特征原子团, 由它们的位置群过渡到商群时不 可约表示的变换, 确定振动模的对称性质.

位置群法便于对振动模分类，应用更普遍. 在 $\mathrm{Al}_{2} \mathrm{BeO}_{4}$ 中, $\mathrm{Be}$ 的地位相当于橄榄石中的 $\mathrm{Si}$ ，四面体 $\mathrm{BeO}_{4}$ 与 $\mathrm{SiO}_{4}$ 相当. 对于正四面体 $\mathrm{BeO}_{4}$ 离子团,具有点群 $T_{d}$, 有四个不同的内振动 模 $A_{1}\left(f_{1}\right), E\left(f_{2}\right), 2 F_{2}\left(f_{3}, f_{4}\right)$, 分别是单重、双重和三重简并态. $f_{1}-f_{4}$ 是相应的频率. 在金 绿宝石中,一个单胞有四个 $\mathrm{BeO}_{4}$ 四面体，具有 $C_{s}$ 对称 ${ }^{[1]}$, 各向异性晶场使其内振动模完全分 裂，商群分解结果为

$$
\begin{aligned}
4 A_{1}\left(f_{1}\right) \rightarrow 4 A^{\prime} \rightarrow A_{g}+B_{2 g}+B_{1 u}+B_{3 u}, \\
4 E\left(f_{2}\right) \rightarrow 4 A^{\prime}+4 A^{\prime \prime} \rightarrow A_{g}+B_{1 g}+B_{2 g}+B_{3 g}+A_{u}+B_{1 u}+B_{2 u}+B_{3 u}, \\
\left.4 F_{2}\left(f_{3}\right)\right\rangle \rightarrow 8\left(2 A^{\prime}+A^{\prime \prime}\right) \rightarrow 2\left(2 A_{g}+B_{1 g}+2 B_{2 g}+B_{3 g}\right. \\
\left.\left.4 F_{2}\left(f_{4}\right)\right\rangle \rightarrow B_{u}+2 B_{1 u}+B_{2 u}+2 B_{3 u}\right) .
\end{aligned}
$$

这里 $A^{\prime}$ 和 $A^{\prime \prime}$ 是位置群的不可约表示. 这里共有 18 支内振动 Raman 活性模, 其中包括 8 支 伸缩模 $\left(f_{1}, f_{3}\right)$, 具有较高的频率, 和 10 支弯曲模 $\left(f_{2}, f_{4}\right)$, 频率比伸缩模低. 在不同对称模 中, 它们的分布情况如下:

$$
\begin{aligned}
& A_{g}: f_{1}+2 f_{3}, f_{2}+2 f_{4}, B_{1 g}: f_{3}, f_{2}+f_{4}, \\
& B_{2 g}: f_{1}+2 f_{3}, f_{2}+2 f_{4}, B_{3 g}: f_{3}, f_{2}+f_{4} .
\end{aligned}
$$

外振动模是由 $\mathrm{BeO}_{4}$ 整体的平动和转动, 以及 $\mathrm{Al}$ 离子的平动引起的. 一个单胞有 4 个 $\mathrm{Al}$ 处于 $\mathrm{Cs}$ 对称位置, 另外 4 个 $\mathrm{Al}$ 处于 $\mathrm{Ci}$ 对称位置. 商群 $D_{2 h}$ 的分解结果为

$$
\Gamma\left(\mathrm{Al}_{s}\right)_{T} \rightarrow 4\left(2 A^{\prime}+A^{\prime \prime}\right) \rightarrow 2 A_{g}+B_{1 g}+2 B_{2 g}+B_{3 g}
$$

本文 1983 年 9 月 16 日收到。 


$$
\begin{aligned}
& +A_{u}+2 B_{1 u}+B_{2 u}+2 B_{3 u}, \\
\Gamma\left(\mathrm{Al}_{i}\right)_{T} \rightarrow & 4\left(3 A_{u}^{\prime}\right) \rightarrow 3 A_{u}+3 B_{1 u}+3 B_{2 u}+3 B_{3 u}, \\
\Gamma\left(\mathrm{BeO}_{4}\right)_{T} & \rightarrow 4\left(2 A^{\prime}+A^{\prime \prime}\right) \rightarrow 2 A_{g}+B_{1 g}+2 B_{2 g}+B_{3 g} \\
& +A_{u}+2 B_{1 u}+B_{2 u}+2 B_{3 u}, \\
\Gamma\left(\mathrm{BeO}_{4}\right)_{R} & \rightarrow 4\left(A^{\prime}+2 A^{\prime \prime}\right) \rightarrow A_{g}+2 B_{1 g}+B_{2 g}+2 B_{3 g} \\
& +2 A_{u}+B_{1 u}+2 B_{2 u}+B_{3 u} .
\end{aligned}
$$

在 Raman 活性的外振动模中 (即晶格模), 转动模共 6 支: $A_{g}+2 B_{1 g}+B_{2 g}+2 B_{3 g}$; 平动模 共 12 支: $4 A_{g}+2 B_{1 g}+4 B_{2 g}+2 B_{3 g}$.

全部振动模为

$$
\Gamma=11 A_{g}+7 B_{1 g}+11 B_{2 g}+7 B_{3 g}+10 A_{u}+14 B_{1 u}+10 B_{2 u}+14 B_{3 u} \text {. }
$$

其中声学模为 $B_{1 u}+B_{2 u}+B_{3 u}$, Raman 活性模共 36 支:

$$
\Gamma_{g}=11 A_{g}+7 B_{1 g}+11 B_{2 g}+7 B_{3 g} \text {. }
$$

红外活性模共 35 支:

$$
\Gamma_{u}=13 B_{1 u}+9 B_{2 u}+13 B_{3 u} .
$$

Raman 和红外都是非活性的模为 $10 A_{u}$. 由 (3) 式可以看出, 处于反演中心的 $\mathrm{Al}$ 离子对 Raman 散射无贡献.

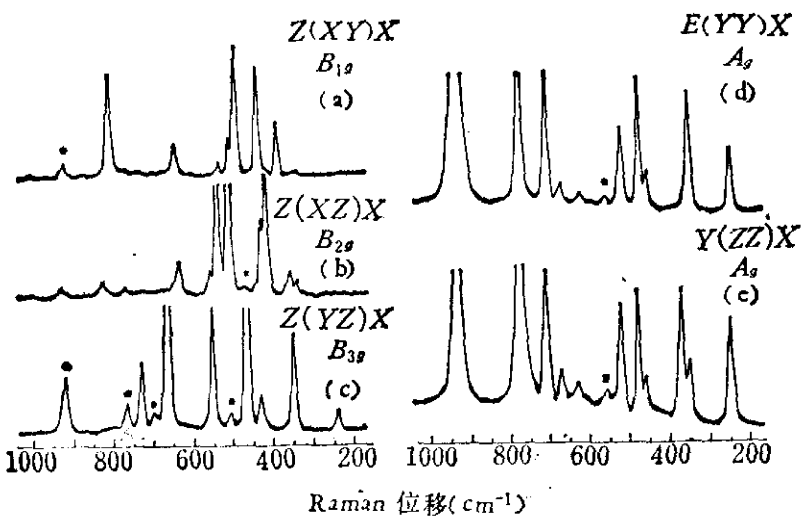

图 1 在 $Z(X Y) X, Z(X Z) X, Z(Y Z) X, Z(Y Y) X$ 和 $Y(Z Z) X$ 配置下 $\mathrm{Al}_{2} \mathrm{BeO}_{4}$ 的 Raman 散射谱(室温) 带*号的峰是泄漏模

商群分析法给出完全相同的结果.

实验用的 $\mathrm{Al}_{2} \mathrm{BeO}_{4}$ 晶体含有 $0.05 \% \mathrm{Cr}^{3+}$ 离子,用提拉法生长.晶体沿 (010) 面切成厚

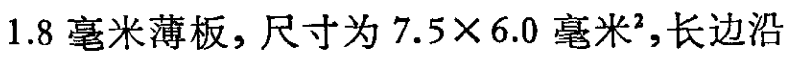
$\langle 100\rangle$ 轴, 短边沿 $\langle 001\rangle$ 轴. 定向偏差约 $2^{\circ}$. 使用氩离子激光 器 4880 埃 线, 功率 100 毫 瓦. 用 HRD-1 双光栅单色仪和光子计数分 析散射光. Raman 位移的测试误差小于 4 $\mathrm{cm}^{-1}$. 检偏器和狭缝之间置有扰偏器, 以消 除光栅对偏振态的衍射效率的差异.

图 $1 \mathrm{a}-\mathrm{d}$ 是四种散射配置 $Z(X Y) X, Z$ $(X Z) X, Z(Y Z) X$ 和 $Z(Y Y) X$ 的室温 Raman 谱,分别相应着 $B_{1 g}, B_{2 g}, B_{3 g}$ 和 $A_{g}$ 对称振动模. 表 1 给出 Raman 位移, 相对强度 和模式的指认情况.

金绿宝石和檄榄石一样,由于对称性较低,单胞分子数目较多,模式的指认相当困难,正则 模的振动状态的分析更不容易. 对于橄榄石和 $\mathrm{Mg}_{2} \mathrm{SiO}_{4}$, 一些研究者曾利用固溶体或同位素 替代的 Raman 谱来分析各振动模的归属, 取得相当好的结果. 由于声子模之间的相互作用和 混合, 许多声子模实际上是混合态,使某些晶格模和内振动模仍然难于区分开来.

和橄榄石相比, $\mathrm{Al}_{2} \mathrm{BeO}_{4}$ 的 Raman 谱中, $\mathrm{BeO}_{4}$ 内振动模的频率偏低, 平移晶格模频率偏 高, 结果, 使内振动模和晶格模的区分更不容易. 参考橄榄石的结果 ${ }^{[2-4]}$, 对于 $\mathrm{Al}_{2} \mathrm{BeO}_{4}$ 振动模 给出以下粗略的指认:

（1）在 703-929 $\mathrm{cm}^{-1}$ 之间的 8 条谱线可能相应于 $\mathrm{BeO}_{4}$ 的伸缩模. 在 $Z(X Y) X$ 配置中, 具有 $B_{1 \mathrm{~g}}$ 对称的 $f_{3}$ 模仅有一支. 实验观测到两条高于 $703 \mathrm{~cm}^{-1}$ 的峰, 根据其峰位置和这里的 
表 1 在 $Z(Y Y) X, Z(X Y) X, Z(X Z) X$ 和 $Z(Y Z) X$ 配置下, $\mathrm{Al}_{2} \mathrm{BeO}_{4}$ 晶体的 Raman 谱频移、相对强度和对称性

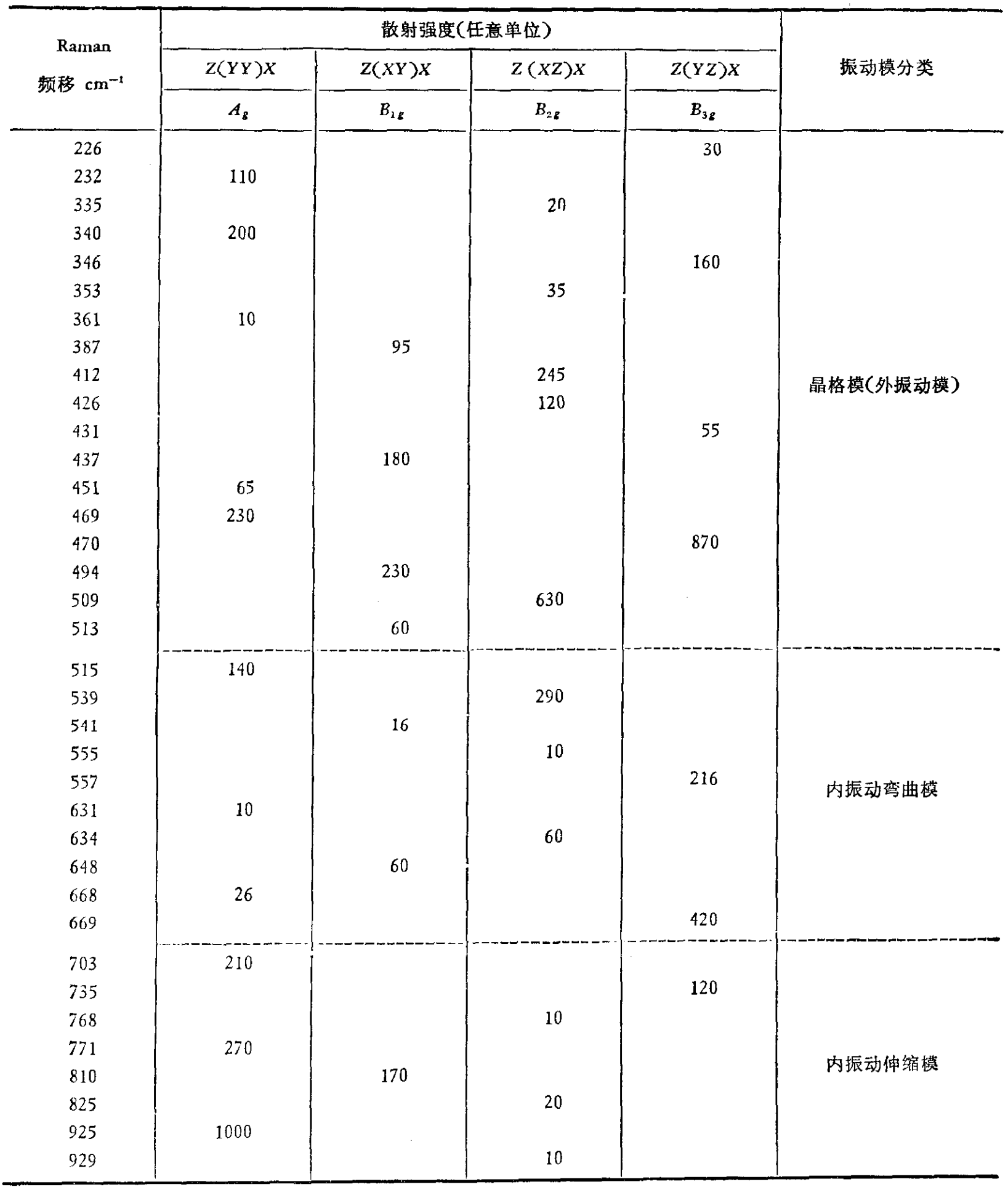

模式分析, 认定 $925 \mathrm{~cm}^{-1}$ 附近的谱线是泄漏峰. 根据同样的理由, 在 $Z(Y Z) X$ 配置的 Raman 谱中, $925,771,703 \mathrm{~cm}^{-1}$ 峰都是泄漏峰.

(2) 在 $515-669 \mathrm{~cm}^{-1}$ 之间的 Raman 谱线可以认为是内振动弯曲模 $f_{2}, f$. 这样的安排 也符合这两组模在各对称态中存在的数目 (见式 (2)).

(3) 在 236- $513 \mathrm{~cm}^{-1}$ 之间的谱被认为是晶格模. 由于振动频率相近, 难于将转动模和平 
动模区分开来.

当激光束沿 $Y$ 轴入射时, 可以得到另外四种配置的 Raman 谱, $Y(X Y) X, Y(X Z) X, Y$ $(Z Y) X$ 和 $Y(Z Z) X$. 前三种配置的 Raman 谱相应出现 $B_{1 g}, B_{2 g}$ 和 $B_{3 g}$ 对称模, 谱的相对 强度和前一组配置基本相同. $Y(Z Z) X$ 配置下出现的 $A_{g}$ 模和 $Z(Y Y) X$ 配置有明显的差 别(图 1e). 在 $Z(Y Y) X$ 配置中, $361 \mathrm{~cm}^{-1}$ 峰十分微弱, 几乎辨认不出来, 而在 $Y(Z Z) X$ 配 置, 却增强了几十倍. 此外, $771 \mathrm{~cm}^{-1}$ 峰也增强了近四倍. 事实上, 像 $361 \mathrm{~cm}^{-1}$ 这样的弱峰, 正 是在等价配置的 Raman 谱中确认下来的.

致谢: 上海光学精密机械研究所马笑山同志提供了 $\mathrm{Al}_{2} \mathrm{BeO}_{4}$ 晶体, 傅全贵同志进行了晶体定向; 曾与顾 本源同志进行过有益的讨论, 在此表示衰心感谢.

\section{参 考 文 献}

[ 1 ] Fartell, E. F., Fang, J. H. and Newnham, R. E., Amer. Mineralogist, 48 (1963), 804.

[ 2 ] Paques-Ledent, M. Th. and Tarte, P., Spectro chimica Acta., 29A (1973), 1007.

[ 3 ] Servoin, J. L. and Piriou, B., Phys. Stat. Sol. (B), 55 (1973), 677.

[4] Iishi, K., Amer. Mineralogist, 63 (1978), 1198.

[ 5 ] Walling, J. C., Peterson, O. G., Jenssen, H. P., Morris, R. C. and O'Dell, E. W., IEEE Quantum Electronics, QE-16 (1980), 1302.

[6] Maradudin, A. A. and Vosko, S. H., Rev. Modern Phys., 40 (1968), 1.

[ 7 ] Poulet, H. and Mathieu, J. P., Vibration Spectra and Symmetry of Crystals, Gordon and Breach, New York, 1976. 